\title{
Pain Outcomes after Platelet-Rich Plasma Application in Chronic Tibialis Anterior Pain: A Retrospective Case Se- RIES ANALYSIS
}

\author{
Calvin Rong Chen, DO'1, Catherine Jameson, DO', Tungming Leung, $\mathrm{PhD}^{2}$, and Steve M. Aydin, DO ${ }^{1,3}$
}

Background: Platelet-rich plasma (PRP) has become a main component of regenerative medicine. It is used in various musculoskeletal tendinous and skeletal injuries, however, literature regarding PRP's use in chronic anterior ankle pain is limited.

Objectives: To determine the effectiveness of PRP in treating tibialis anterior (TA) tendon pain in patients who have failed traditional conventional therapeutic treatments.

Study Design: Retrospective chart review.

Setting: Outpatient interventional pain clinic at an academic center.

Methods: Adults $(n=10)$, aged 18 and older, with greater than 6 months of TA tendon pain who failed conservative therapies. Patients who had undergone previous regenerative injections were excluded. PRP injection was administered with ultrasound guidance. Change in pain score was assessed using the Numeric Rating Scale (NRS-11: 0 = no pain, 10 = excruciating pain), and patient's self-reported reduction in pain (percentage), pre- and postprocedure. There was a reduction in opioid dosage postprocedure. Adverse effects were recorded.

Results: Average pain score at baseline was 8 \pm 1.5 on the NRS-11 scale. The average percent change in pain between baseline and 8 weeks posttreatment was a $36 \% \pm 23.2 \%$ reduction in pain. This was a significant reduction in pain score at 8 weeks post-PRP treatment when compared with baseline, $P=0.008$. Average reduction in opioid dose between baseline and 8 weeks posttreatment was $17 \% \pm 21.2 \%, P=0.063$. No patients experienced adverse effects.

Limitations: Small sample size, and no doubleblind randomization with control group.

Conclusions: PRP use in chronic TA pain 8 weeks post-PRP treatment significantly improved chronic TA pain. There is also a tendency toward lower opioid pain medication dosages after 8 weeks post-PRP treatment, although this was not statistically significant.

Key words: Platelet-rich plasma, regenerative medicine, pain, ultrasound
From : 'Department of Physical Medicine and Rehabilitation, Donald and Barbara Zucker School of Medicine at Hofstra-Northwell, Manhasset, NY; ${ }^{2}$ Department of Biostatistics, Northwell Health, Manhasset, NY; ${ }^{3}$ Kayal Orthopaedic Center, P.C., Bergen County, NJ

Author for correspondence: Calvin Rong Chen, DO

Address: Department of Physical Medicine and Rehabilitation, Donald and Barbara Zucker School of Medicine at Hofstra-Northwell, 1554 Northern Blvd, 4th Floor PM\&R, Manhasset, NY 11030

E-mail: cchen00723@gmail.com

Disclaimer: There was no external funding in the preparation of this manuscript. Conflict of interest: Each author certifies that he or she, or a member of his or her immediate family, has no commercial association (i.e., consultancies, stock ownership, equity interest, patent/licensing arrangements, etc.) that might pose a conflict of interest in connection with the submitted manuscript.
It is estimated that roughly half of all lower extremity injuries are due to overuse injuries. Overuse, seen in both recreational and professional athletes, involves frequent repetitive movements that cause microtraumatic tendinopathies (1). Chronic ankle pain, specifically, commonly stems from biomechanical and anatomic variations, as well as postsurgical changes after orthopedic repair. Conservative therapies consisting of rest, ice, compression, and elevation (also called RICE), and conventional physiotherapy can improve repetitive ankle muscle strain and correct chronic muscle imbalances. In some severe circumstances, surgical intervention is required to treat ankle joint instability or tendon/ligament rupture. However, 
there is no current consensus within the medical community regarding how best to speed recovery of these injuries (2).

Over the last decade, regenerative medicine has worked its way into the armamentarium of practicing physicians. Foremost among options is platelet-rich plasma (PRP), which has gained traction as a treatment method for various musculoskeletal conditions based on its safety profile and its potential ability to enhance the body's own ability to promote soft-tissue healing (3).

PRP is an autologous biologic agent postulated to expedite tissue healing through the injection of growth factors and cytokines into areas of damaged tissue $(4,5)$. Patient blood is centrifuged, isolating platelets and leukocytes from red blood cells. Significant variations exist among preparation methods and platelet concentrations in the final PRP product, and activating agents (e.g., thrombin, calcium) may also be added to enhance the release of growth factors. Alternatively, "inactivated PRP" may be used, becoming activated on injection and exposure to collagen and thromboplastin (6). Once activated, platelets release several growth factors, including plateletderived growth factor, vascular endothelial growth factor, and epidermal growth factor (5). These factors, when applied to damaged tissue, potentially promote angiogenesis and aid in the localization, propagation, and differentiation of stem cells. The end result of this cascade is improved healing of damaged tissues (6).

PRP has been shown in vitro to enhance fibrocyte and collagen proliferation (7). Further, studies have shown that it's in vivo application promotes healing and increases tensile strength in tendons that have undergone traumatic injury (7). A review of studies on PRP's effectiveness in tendinopathies also found decreased tendon repair time, increased fiber organization, or both with PRP treatment through improving the metabolic functions of damaged tissues (8). Despite these observations, the exact mechanism behind PRP's efficacy has yet to be elucidated $(8,9)$.

To date, the potential for pain reduction and improved functionality in patients enduring lower extremity injuries have been promising (10). PRP's ever-increasing popularity among physicians can be attributed to its relatively simple and swift preparation and ease of administration, particularly under ultrasound guidance. The incidence of PRP's adverse effects are likely limited due to PRP's autologous nature and the accuracy of local administration to the desired area, minimizing the potential for drug interactions (11).

PRP has become a mainstay of regenerative medicine. Despite PRP's ease of use, minimal side effect profile, and reputation as a relatively safe procedure to treat a wide spectrum of musculoskeletal conditions, there remain few studies in the current literature focusing on patients' opioid medication reduction after PRP injections. The aim of this retrospective study was to determine the effectiveness of PRP in treating chronic tibialis anterior (TA) tendon ankle pain by examining the differences in pain and opioid dosages between baseline and 8 weeks post-PRP treatment. Here we examine a series of 10 cases of reported pain over the ankle and TA tendon, all of which had previously failed traditional conventional therapeutic treatments.

\section{METHODS}

This study was a retrospective chart review of 10 patient cases of male and female patients over the age of 18. Patients were required to have ankle pain along the anterior tibialis tendon or muscle with symptoms (pain) lasting for greater than 6 months. Patients must also have failed conservative therapies with no long-term improvement, and those who had previously received regenerative injections were excluded. Any surgical interventions pertaining to the ankle pain were recorded. The patients also underwent prescreen complete blood count to ensure an adequate level of platelets. Cessation of aspirin, steroids, or nonsteroidal antiinflammatory medications 2 weeks prior to the procedure and 3 weeks after the procedure was also required.

The PRP venipuncture was performed under sterile technique with an $18 \mathrm{G}$ needle. The first $5 \mathrm{~mL}$ of blood was discarded, and an additional $40 \mathrm{~mL}$ of blood was removed and mixed with $5 \mathrm{~mL}$ of dextrose citrate in the syringe for a total of $45 \mathrm{~mL}$ of fluid. The sample was placed in a centrifuge using the Arteriocyte system for 19 minutes, as shown in Fig. 1. A total of $4 \mathrm{~mL}$ of PRP was obtained. The test patients' TA 


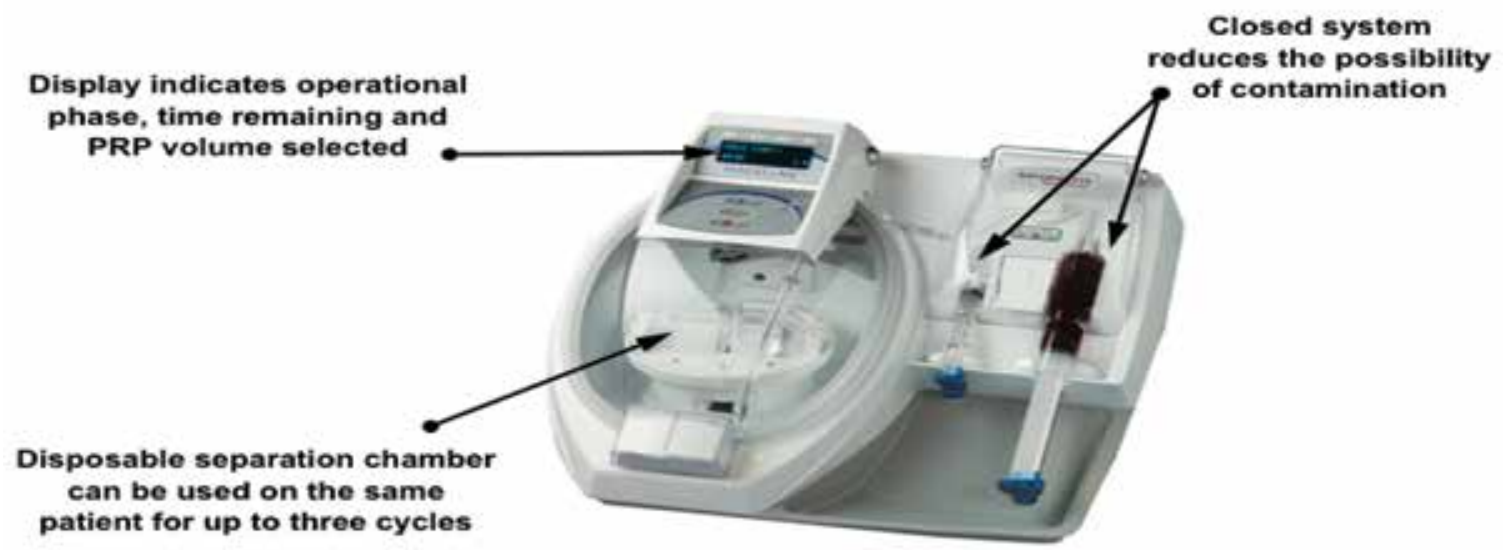

Fig. 1. Arteriocyte system for PRP preparation.

tendons were first identified with an ultrasound in short axis, as illustrated in Figs. 2 and 3. The injection of PRP was performed under sterile technique using live ultrasound. The injectate was placed along the distal insertion of the TA tendon with a 25G 1.5-inch needle and $5 \mathrm{~mL}$ syringe and was identified on live ultrasound, as shown in Fig. 4. This procedure was performed without local anesthetic or other agents to maintain a neutral $\mathrm{pH}$ environment.

Patients were asked to give a percent change in pain before the PRP injection and 8 weeks after the PRP treatment via the Numeric Rating Scale (NRS-11). In patients taking opioid medications, the change in opioid dosages was calculated based on the opioid dosages before and 8 weeks after the PRP treatment.

\section{RESULTS}

All patients received the PRP injection at the same site, anterior tibialis tendon, with the Arteriocyte PRP system. Only one patient was not on opioid medications during the study. All the patients reported pain at baseline. The average pain score at baseline was $8 \pm 1.5$ on the NRS-11 scale of 0 to 10 , with 10 indicating the highest level of pain. The average percent reduction in pain between baseline and 8 weeks posttreatment was $36 \% \pm 23.2 \%$. Of the 10 patients, only 1 reported no $(0 \%)$ change in pain after 8 weeks of PRP treatment. The remaining 9 patients reported reduced pain. None of the patients reported that the pain was worse at 8 weeks post-PRP treatment when compared with baseline (Table 1).

The average opioid dose reduction between baseline and 8 weeks posttreatment was $17 \% \pm 21.2 \%$. Three of the patients did not change their opioid dose after treatment with PRP. There was no significant change in opioid dose after 8 weeks post-PRP treatment in this study when compared with baseline, $P$ $=0.063$ (Table 2).

\section{DISCUSSION}

Our data indicate that PRP may have a role providing pain relief to those affected by chronic TA pain. This is consistent with the current beliefs regarding the mechanism by which PRP promotes healing in damaged tissues, namely by injection of growth factors and cytokines into areas of damaged tissue. More specifically, using PRP as a therapeutic modality for musculoskeletal ailments uses the known physiological inflammatory response and results in enhanced recruitment of various immune cells essential for increased myogenesis (12). This may be responsible for our study's improved pain relief during the patients' postinjection follow-ups.

Our findings also suggest that PRP may provide sufficient pain relief such that patients are able to decrease their dosages of opioid pain medications. Although not statistically significant, our results suggested a tendency toward lower opioid pain medication dosages at 8 weeks after PRP treatment. 

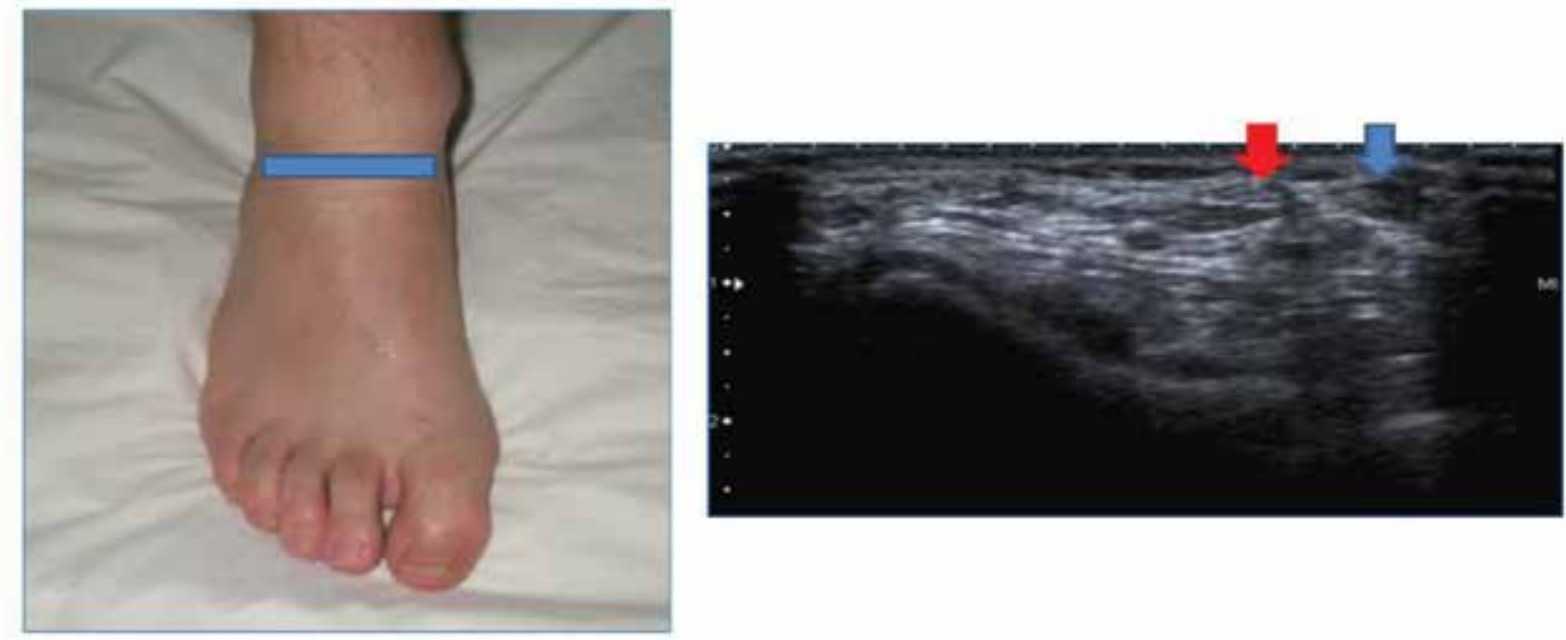

Fig. 2. Anatomy of the TA under evaluation with ultrasound.

(a)

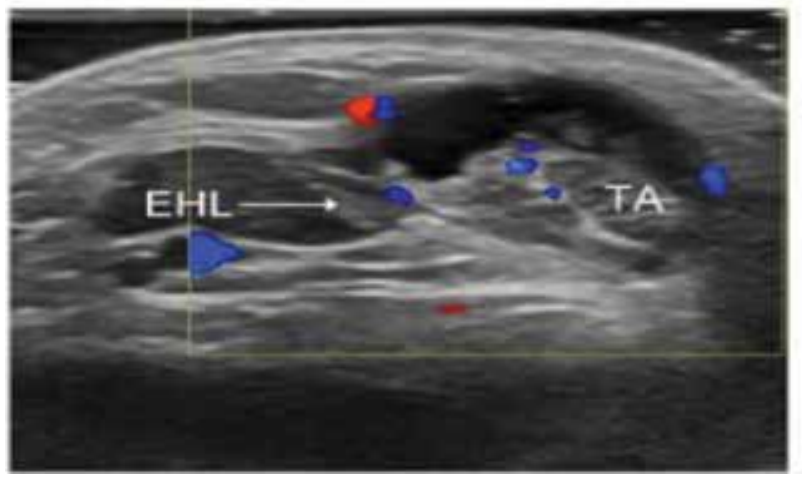

(b)

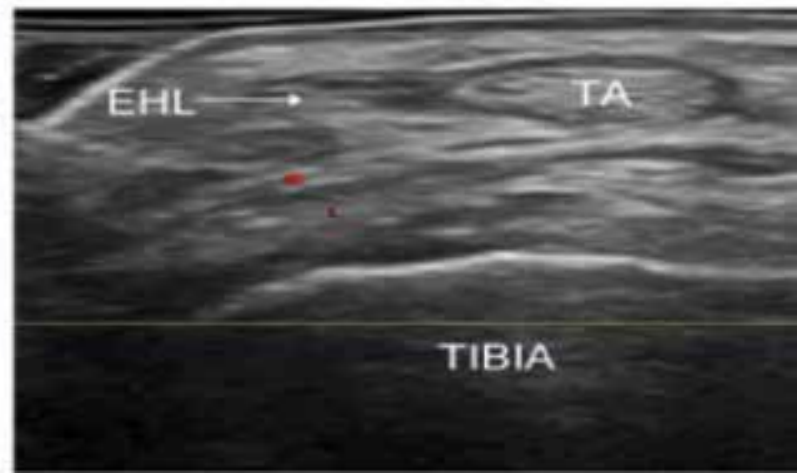

Fig. 3. Short axis TA views: (A) TA with swell, (B) normal TA and extensor hallucis longus tendon sheaths.
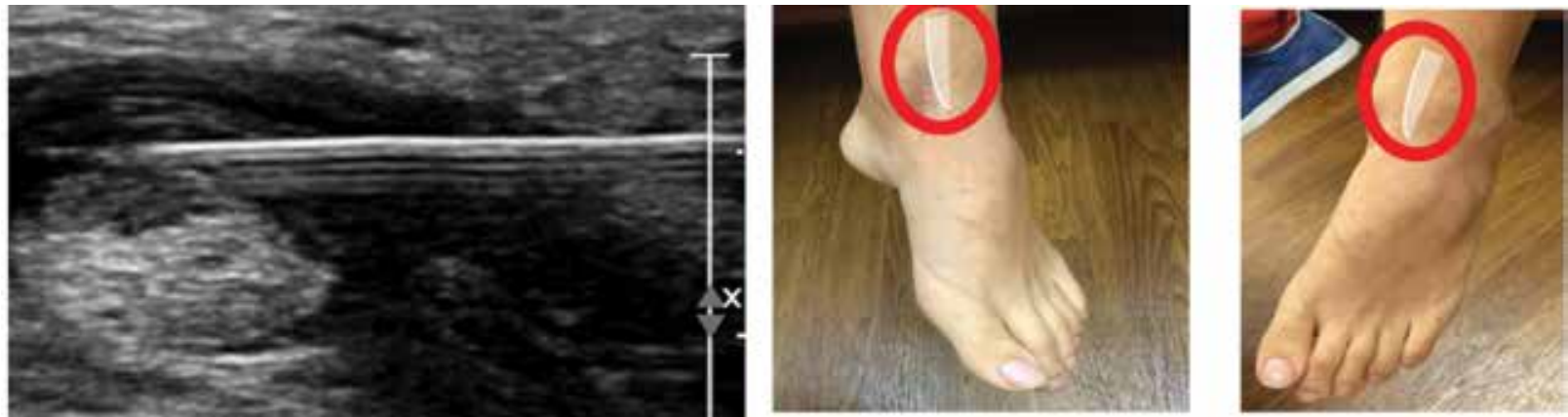

Fig. 4. PRP injection of the TA tendon and sheath. Left: ultrasound-guided injection of PRP, middle and right: ankle with markings of the TA tendon injection sites. 
Our study is incongruent with a recent 2019 study looking at PRP injections with varying leukocytic formulations in the treatment of patellar tendinopathy in which there was no statistically significant improvement in pain symptoms between the PRP groups and the group injected with saline solution (13). Most PRP studies focusing on musculoskeletal injuries have promising, but varied, findings. Because of its autologous profile, PRP's risk profile remains low and it is a relatively safe adjunct to pharmacologic pain management in tendinopathies.

\section{CONCLUSIONS}

PRP has become an increasingly popular treatment for chronic musculoskeletal pain among clinicians. Our pilot study supports its use in the management of chronic ankle pain due to underlying tendon damage, but given our limited sample size, a larger
Table 1. NRS-11 pain scores at baseline and percent improvement in pain after PRP injection.

\begin{tabular}{|l|c|c|c|c|c|}
\hline & Mean & $\begin{array}{c}\text { Standard } \\
\text { Deviation }\end{array}$ & Median & Minimum & Maximum \\
\hline $\begin{array}{l}\text { Initial NRS-11 } \\
\text { pain score (0-10) }\end{array}$ & 7.89 & 1.54 & 8.00 & 6.00 & 10.00 \\
\hline $\begin{array}{l}\text { Pain } \\
\text { improvement (\%) }\end{array}$ & 35.60 & 23.20 & 35.00 & 0.00 & 75.00 \\
\hline
\end{tabular}

Table 2. Pain reduction in opioid medications post-PRP injection.

\begin{tabular}{|l|l|l|l|l|l|}
\hline & Mean & $\begin{array}{l}\text { Standard } \\
\text { Deviation }\end{array}$ & Median & Minimum & Maximum \\
\hline $\begin{array}{l}\text { Pain reduction in } \\
\text { opioid medications } \\
\text { after injection (\%) }\end{array}$ & 16.9 & 21.2 & 10.0 & 0.0 & 50.0 \\
\hline
\end{tabular}

case series would be useful. Furthermore, a randomized, doubleblinded study, along with a larger study group, would be valuable in both solidifying our findings and for examining the true efficacy and value of PRP, especially in the reduction of pain medications use in chronic ankle pain.

\section{REFERENCES}

1. Wilder P, Sethi S. Overuse injuries: Tendinopathies, stress fractures, compartment syndrome, and shin splints. Clin Sports Med 2004; 23:55-81.

2. Talaie T, Pratt SJP, Vanegas C, et al. Site-specific targeting of platelet-rich plasma via superparamagnetic nanoparticles. Orthop J Sports Med 2015; 3:2325967114566185.

3. Mlynarek RA, Kuhn AW, Bedi A. Platelet-rich plasma (PRP) in orthopedic sports medicine. Am J Orthop (Belle Mead NJ) 2016; 45:290-326.

4. Magnussen RA, Flanigan DC, Pedroza AD, Heinlein KA, Kaeding $C C$. Platelet rich plasma use in allograft $A C L$ reconstructions: Two-year clinical results of a MOON cohort study. Knee 2012; 20:277-280.

5. Etulain J, Mena HA, Meiss RP, et al. An optimised protocol for platelet-rich plasma preparation to improve its angiogenic and regenerative properties. Sci Rep 2018; 8:1513.

6. Setayesh K, Villarreal A, Gottschalk A, Tokish JM, Choate WS. Treatment of muscle injuries with platelet-rich plasma: A review of the literature. Curr Rev Musculoskelet Med 2018; 11:635-642.

7. Mishra A, Woodall J Jr, Vieira A. Treatment of tendon and mus- cle using platelet-rich plasma. Clin Sports Med 2009; 28:113125.

8. Zhou Y, Wang JH. PRP Treatment efficacy for tendinopathy: A review of basic science studies. Biomed Res Int 2016; 2016:9103792.

9. de Mos $M$, van der Windt $A E$, Jahr $H$, et al. Can platelet-rich plasma enhance tendon repair? A cell culture study. Am J Sports Med 2008; 36:1171-1178.

10. Engebretsen L, Steffen K, Alsousou J, et al. IOC consensus paper on the use of platelet-rich plasma in sports medicine. $\mathrm{Br} \mathrm{J}$ Sports Med 2010; 44:1072-1081.

11. Laver L, Marom N, Dnyanesh L, Mei-Dan O, EspregueiraMendes J, Gobbi A. PRP for degenerative cartilage disease: A systematic review of clinical studies. Cartilage 2017; 8:341-364.

12. Andia I, Abate M. Platelet-rich plasma in the treatment of skeletal muscle injuries. Expert Opin Biol Ther 2015; 15:987-999.

13. Scott A, LaPrade RF, Harmon KG, et al. Platelet-rich plasma for patellar tendinopathy: A randomized controlled trial of leukocyte-rich PRP or leukocyte-poor PRP versus saline. Am J Sports Med 2019; 47:1654-1661. 
IPM Reports 